\title{
HIGHER ORDER GENOCCHI, EULER POLYNOMIALS ASSOCIATED WITH $q$-BERNSTEIN TYPE POLYNOMIALS
}

\author{
Serkan Araci And Dilek Erdal
}

\begin{abstract}
The main aim of this paper is to give some relationships between $q$-Bernstein, higher order genocchi and Euler polynomials.
\end{abstract}

\section{Introduction, Definitions and Notations}

Throughout this paper, $\mathbb{C}$ denotes the complex number field and $\mathbb{N}$ the set of natural numbers. We assume that $q \in \mathbb{C}$ with $|q|<1$ and that the $q$-number is defined by $[x]_{q}=\frac{q^{x}-1}{q-1}$ (see [1],[2],[5]-[18]). The generating functions of the higher order Genocchi , Euler polynomials and $q$-Bernstein polynomials, respectively, can be defined as follows:

$$
\begin{aligned}
& D^{(w)}(x, t)=\left(\frac{2 t}{e^{t}+1}\right)^{w} e^{x t}=\sum_{n=0}^{\infty} G_{n}^{(w)}(x) \frac{t^{n}}{n !}, \text { for }|t|<\pi, \\
& S^{(w)}(x, t)=\left(\frac{2}{e^{t}+1}\right)^{w} e^{x t}=\sum_{n=0}^{\infty} E_{n}^{(w)}(x) \frac{t^{n}}{n !}, \text { for }|t|<\pi
\end{aligned}
$$

(see [13],[18],[19]) and

$F_{k}(x, t ; q)=\frac{\left(t[x]_{q}\right)^{k}}{k !} e^{t[1-x]_{q}}=\sum_{n=k}^{\infty} B_{k, n}(x ; q) \frac{t^{n}}{n !}, t \in \mathbb{C}, k=0,1, \cdots, n$.

where $\lim _{q \rightarrow 1} F_{k}(x, t ; q)=F_{k}(t, x)=\frac{(t x)^{k}}{k !} e^{t(1-x)}$ (see [3]).

Received March 6, 2011. Accepted March 22, 2011.

2000 Mathematics Subject Classification. 05A10, 11B65, 28B99, 11B68, 11B73.

Key words and phrases. Generating function, Higher order Euler polynomials, Higher order Genocchi polynomials, Mellin transformation, Zeta function, $q$-Bernstein polynomials. 
By (1.1) and (1.2) we easily see that

$$
D^{(w)}(x, t)=t^{w} S^{(w)}(x, t)
$$

From the above, we have

$$
\sum_{n=0}^{\infty} G_{n}^{(w)}(x) \frac{t^{n}}{n !}=\sum_{n=0}^{\infty} E_{n}^{(w)}(x) \frac{t^{n+w}}{n !}
$$

By comparing coefficients $t^{n}$ in the both sides of the above equation for $n \in \mathbb{N}$,

$$
G_{n+w}^{(w)}(x)=\frac{(n+w) !}{(n) !} E_{n}^{(w)}(x)
$$

These polynomials have explicit formulas, respectively, as follows:

$$
\begin{gathered}
G_{n}^{(w)}(x)=\sum_{k=0}^{n}\left(\begin{array}{l}
n \\
k
\end{array}\right) G_{k}^{(w)} x^{n-k} \\
E_{n}^{(w)}(x)=\sum_{k=0}^{n}\left(\begin{array}{l}
n \\
k
\end{array}\right) E_{k}^{(w)} x^{n-k} \\
B_{k, n}(x ; q)=\left(\begin{array}{l}
n \\
k
\end{array}\right)[x]_{q}^{k}[1-x]_{q}^{n-k}, x \in[0,1]
\end{gathered}
$$

where $G_{k}^{(w)}=G_{k}^{(w)}(0), E_{k}^{(w)}=E_{k}^{(w)}(0)$ are higher order Genocchi and Euler numbers, respectively.

The purpose of this paper is to give some relationships between $q$ Bernstein, higher order Genocchi and Euler polynomials. We derive the higher order zeta functions from the Mellin transformation of this generating function which interpolates the higher order Genocchi polynomials at negative integers and associated with $q$-Bernstein polynomials. Furthermore, we define particular higher order zeta functions associated with $q$-Bernstein polynomials.

\section{New Identities On $q$-Bernstein Type Polynomials}

Theorem 1. Let $n \in \mathbb{N}$ and $0 \leq w \leq n$. We obtain

$$
B_{w, n}(x ; q)=\frac{[x]_{q}^{w}}{2^{n-w} w !} \sum_{l=0}^{n}\left(\begin{array}{l}
n \\
l
\end{array}\right) G_{l}^{(w)}\left(2[1-x]_{q}\right) E_{n-l}^{(-w)} .
$$


Proof. By using (1.1),(1.2) and (1.3) we have

$$
\begin{aligned}
& \sum_{n=k}^{\infty} 2^{n} B_{w, n}(x ; q) \frac{t^{n}}{n !} \\
= & \frac{\left(2[x]_{q}\right)^{w}}{w !}\left(\sum_{n=0}^{\infty} G_{n}^{(w)}\left(2[1-x]_{q}\right) \frac{t^{n}}{n !}\right)\left(\sum_{n=0}^{\infty} E_{n}^{(-w)} \frac{t^{n}}{n !}\right)
\end{aligned}
$$

By using Cauchy product in the above we have

$$
\begin{aligned}
& \sum_{n=w}^{\infty} 2^{n} B_{w, n}(x ; q) \frac{t^{n}}{n !} \\
= & \frac{\left(2[x]_{q}\right)^{w}}{w !} \sum_{n=0}^{\infty}\left(\sum_{l=0}^{n}\left(\begin{array}{l}
n \\
l
\end{array}\right) G_{l}^{(w)}\left(2[1-x]_{q}\right) E_{n-l}^{(-w)}\right) \frac{t^{n}}{n !}
\end{aligned}
$$

From the above we have

$$
\begin{aligned}
& \sum_{n=w}^{\infty} 2^{n} B_{w, n}(x ; q) \frac{t^{n}}{n !} \\
= & \frac{\left(2[x]_{q}\right)^{w}}{w !} \sum_{n=0}^{w-1}\left(\sum_{l=0}^{n}\left(\begin{array}{l}
n \\
l
\end{array}\right) G_{l}^{(w)}\left(2[1-x]_{q}\right) E_{n-l}^{(-w)}\right) \frac{t^{n}}{n !} \\
& +\frac{\left(2[x]_{q}\right)^{w}}{w !} \sum_{n=w}^{\infty}\left(\sum_{l=0}^{n}\left(\begin{array}{l}
n \\
l
\end{array}\right) G_{l}^{(w)}\left(2[1-x]_{q}\right) E_{n-l}^{(-w)}\right) \frac{t^{n}}{n !}
\end{aligned}
$$

By comparing coefficients of $\frac{t^{n}}{n !}$ in the both sides of the above equation, we arrive at the desired result.

From (2.2), we get the following corollary:

Corollary 1. Let $w \in \mathbb{N}$ with $w \leq n$. Then we have

$$
\frac{\left(2[x]_{q}\right)^{w}}{w !} \sum_{n=0}^{w-1} \sum_{l=0}^{n}\left(\begin{array}{l}
n \\
l
\end{array}\right) G_{l}^{(w)}\left(2[1-x]_{q}\right) E_{n-l}^{(-w)}=0 .
$$


From (1.1), we have

$$
\begin{aligned}
D^{(w)}(t, x) & =\left(\frac{2 t}{e^{t}+1}\right) \cdot\left(\frac{2 t}{e^{t}+1}\right) \ldots\left(\frac{2 t}{e^{t}+1}\right) e^{t x} \\
& =2^{w} t^{w} e^{x t} \sum_{n_{1}=0}^{\infty}(-1)^{n_{1}} e^{n_{1} t} \sum_{n_{2}=0}^{\infty}(-1)^{n_{2}} e^{n_{2} t} \ldots \sum_{n_{w}=0}^{\infty}(-1)^{n_{w}} e^{n_{w} t} \\
& =2^{w} t^{w} \sum_{n_{1}, n_{2}, \ldots, n_{w=0}}^{\infty}(-1)^{n_{1}+n_{2}+\ldots+n_{w}} e^{\left(x+n_{1}+n_{2}+\ldots+n_{w}\right) t} \\
& =\sum_{n=0}^{\infty} G_{n}^{(w)}(x) \frac{t^{n}}{n !} .
\end{aligned}
$$

By using (2.4) we easily see that,

$$
\begin{aligned}
& (2.5) G_{n+w}^{(w)}(x) \\
& =2^{w} \frac{(n+w) !}{n !} \sum_{n_{1}, n_{2}, \ldots, n_{w=0}}^{\infty}(-1)^{n_{1}+n_{2}+\ldots+n_{w}}\left(x+n_{1}+n_{2}+\ldots+n_{w}\right)^{n} .
\end{aligned}
$$

For $s \in \mathbb{C}$ we have

$$
\begin{aligned}
& \frac{1}{\Gamma(s)} \int_{0}^{\infty} D^{(w)}(-t, x) t^{s-w-1} d t \\
= & 2^{w} \sum_{n_{1}, \ldots, n_{w}=0}^{\infty}(-1)^{n_{1}+n_{2}+\ldots+n_{w}} \frac{1}{\Gamma(s)} \int_{0}^{\infty} e^{-\left(x+n_{1}+n_{2}+\ldots+n_{w}\right) t} t^{s-1} d t \\
(2.6)= & 2^{w} \sum_{n_{1}, \ldots, n_{w}=0}^{\infty} \frac{(-1)^{n_{1}+n_{2}+\ldots+n_{w}}}{\left(x+n_{1}+n_{2}+\ldots+n_{w}\right)^{s}}
\end{aligned}
$$

We define zeta function as follows:

$$
\zeta^{(w)}(s, x)=2^{w} \sum_{n_{1}, \ldots, n_{w}=0}^{\infty} \frac{(-1)^{n_{1}+n_{2}+\ldots+n_{w}}}{\left(x+n_{1}+n_{2}+\ldots+n_{w}\right)^{s}} \text { for } \Re(s)>0 \text {. (see [7]) }
$$

Note that $\zeta^{(w)}(s, x)$ can be continued analytically to an entire function of $s \in \mathbb{C}$. By substituting $s=-n$ into (2.5), we obtain (2.5) as follows:

$$
\zeta^{(w)}(-n, x)=\frac{n !}{(n+w) !} G_{n+w}^{(w)}(x), \quad(\text { see }[7])
$$


$\zeta^{(w)}(s, 1)=\zeta^{(w)}(s)$ which is the zeta function, From (1.6) and (2.8) for $x=0$. We can easily derive the following equation:

$$
\zeta^{(w)}(-n)=\frac{n !}{(n+w) !} G_{n+w}^{(w)}=E_{n}^{(w)}
$$

By using (2.1) and (2.9) we get following theorem:

Theorem 2. Let $w \in \mathbb{N}$ with $w \leq n$. Then we have

$$
=\frac{2^{w-n}[x]_{q}^{w}}{w !} \sum_{l=0}^{w}\left(\begin{array}{l}
n \\
l
\end{array}\right) \frac{l !}{(n-l) !} \zeta^{(w)}\left(w-l, 2[1-x]_{q}\right) \zeta^{(-w)}(l-n) .
$$

we define particular zeta function as follows:

$$
\begin{aligned}
H^{(w, \lambda)}\left(s\left|a_{1}, a_{2}, \ldots, a_{w}\right| F\right) & =\sum_{n_{i}=a_{i}(\bmod F)} \frac{\lambda^{n_{1}+n_{2}+\ldots+n_{w}}}{\left(n_{1}+n_{2}+\ldots+n_{w}\right)^{s}}, i=1,2, \ldots, w \\
& =\sum_{n_{i}=0}^{\infty} \frac{\lambda^{\sum_{l=1}^{w} n_{l} F+a_{l}}}{\left(\sum_{l=1}^{w} n_{l} F+a_{l}\right)^{s}} \\
& =\frac{\lambda^{\sum_{l=1}^{w} a_{l}}}{F^{s}} \sum_{n_{i}=0}^{\infty} \frac{\lambda^{F \sum_{l=1}^{w} n_{l}}}{\left(\sum_{l=1}^{w} n_{l}+\frac{a_{l}}{F}\right)^{s}}
\end{aligned}
$$

where $F \equiv 1(\bmod 2)$, by substituting $\lambda=-1$ into $(2.11)$, we have

$$
H^{(w,-1)}\left(s\left|a_{1}, a_{2}, \ldots, a_{w}\right| F\right)=\frac{(-1)^{\sum_{l=1}^{w} a_{l}}}{F^{s} 2^{w}} \zeta^{(w)}\left(s, \frac{\sum_{l=1}^{w} a_{w}}{F}\right)
$$

By using (2.12) we obtain the following theorem:

Theorem 3. Let $n \in \mathbb{N}$ and $F \equiv 1(\bmod 2)$ with $0 \leq a_{i}<F$ for $i=1,2, \ldots, w$. We have

$$
\begin{aligned}
& G_{n+w}^{(w)}\left(\frac{a_{1}+a_{2}+\ldots+a_{w}}{F}\right) \\
= & \frac{(-1)^{\sum_{l=1}^{w} a_{l}}(n+w) ! 2^{w}}{n ! F^{n}} H^{(w,-1)}\left(-n\left|a_{1}, a_{2}, \ldots, a_{w}\right| F\right)
\end{aligned}
$$

From (2.10), (2.12) and (2.13) we get following theorem: 
Theorem 4. Let $F \equiv 1(\bmod 2), n \in \mathbb{N}$ and $0 \leq w \leq n$. One has

$$
\begin{aligned}
& B_{w, n}\left(\frac{\sum_{k=1}^{w} a_{k}}{F} ; q\right)=\frac{2^{2 w-n}(-1)^{\sum_{k=1}^{w} a_{k}}}{w !}\left[\frac{a_{1}+a_{2}+\ldots+a_{w}}{F}\right]_{q} \\
& \times \sum_{l=0}^{n}\left(\begin{array}{c}
n \\
n-l, n-l
\end{array}\right) F^{w-l} H^{(w,-1)} \\
& \times\left(w-l\left|\frac{1}{F}, \frac{q}{F}, \frac{q^{2}}{F}, \ldots, \frac{q^{-\frac{a_{1}+a_{2}+\ldots+a_{w}}{F}}}{F}\right| F\right) \zeta^{(-w)}(l-n) .
\end{aligned}
$$

where $\left(\begin{array}{c}n \\ n-l, n-l\end{array}\right)=\frac{n !}{(n-l) !(n-l) !}$.

\section{References}

[1] M. Açıkgöz, and Y. Şimşek, A New generating function of $q$-Bernstein type polynomials and their interpolation function, Abstract and Applied Analysis, Article ID 769095, 12 pages, doi: 10.1155/2010/769095.01-313.

[2] M. Açıkgöz, S. Aracı, and İ. N.Cangül, A Note on the modified $q$-Bernstein polynomials for functions of several variables and their reflections on $q$-Volkenborn integration, Applied Mathematics and Computation(in press)

[3] M. Açıkgöz, and S. Aracı, On the generating function of Bernstein polynomials, in proceedings of the 8th International conference of Numerical Analysis and Applied Mathematics (ICNAAM 2010), AIP, Rhodes, Greece, March 2010.

[4] L. C. Carlitz, q-Bernoulli numbers and polynomials, Duke Math. J. 15(1948), 987-1000.

[5] I. N. Cangul, V. Kurt, H. Ozden, Y. Simsek, On the higher-order $w$-q-Genocchi numbers, Adv. Stud. Contemp. Math. 19 (2009), 39-57

[6] J. Choi, D. S. Jang and H. M. Srivastava, A generalization of the Hurwitz-Lerch Zeta function, Integral Transforms Spec. Funct. 19 (2008), 65-79.

[7] J. Choi and H. M. Srivastava, The multiple Hurwitz Zeta function and the multiple Hurwitz-Euler Eta function, Taiwanese J. Math. 15 (2011), in press

[8] V. Kac, P. Cheung, Quantum Calculus. Springer, 128 , 2001.

[9] T. Kim, A New Approach to q-Zeta Function, Adv. Stud. Contemp. Math. 11 (2) 157-162.

[10] T. Kim, On the q-extension of Euler and Genocchi numbers, J. Math. Anal. Appl. 326 (2007) 1458-1465.

[11] T. Kim, On the multiple $q$-Genocchi and Euler numbers, Russian J. Math. Phys. 15 (4) (2008) 481-486. arXiv:0801.0978v1 [math.NT]

[12] T. Kim, A Note on the $q$-Genocchi Numbers and Polynomials, Journal of Inequalities and Applications 2007 (2007) doi:10.1155/2007/71452. Article ID 71452, 8.

[13] C-S. Ryoo, T. Kim, B. Lee, and J. Choi, On the generalized q-Genocchi numbers and polynomials of higher order, Advances in Difference Equations, Volume 2011, Article ID 424809, 8page, doi: 10.1155/2011/424809

[14] T. Kim, On a p-adic interpolation function for the q-extension of the generalized Bernoulli polynomials and its derivative, Discrete Mathematics, 309(6) (2009), 1593-1602. 
[15] T. Kim, On p-adic q-l-functions and sums of powers, Journal of Mathematical Analysis and Applications, 329(2) (2007), 1472-1481.

[16] T. Kim, On p-adic q-L-functions and sums of powers, Discrete Mathematics, 252 (2002), 179-187.

[17] T. Kim, On a q-Analogue of thep-Adic Log Gamma Functions and Related Integrals, Journal of Number Theory, 76(2) (1999), 320-329.

[18] Q.-M. Luo, $q$-Extensions for the Apostol-Genocchi Polynomials, General Mathematics, 17(2) (2009), 113-125

[19] Q.-M. Luo, Apostol-Euler Polynomials of Higher Order and Gaussian Hypergeometric Functions, Tanwanse Journal of Mathematics, 10 (4) (2006), 917-925.

\section{Serkan Aracı}

Department of Mathematics, Faculty of Science and Arts, University of Gaziantep,

27310 Gaziantep, Turkey.

E-mail: mtsrkn@hotmail.com

Dilek Erdal

Department of Mathematics, Faculty of Science and Arts, University of Gaziantep, 27310 Gaziantep, Turkey.

E-mail: dilekvecii@mynet.com 